\title{
Efeito da suplementação de probióticos no diabetes mellitus: uma revisão sistemática
}

\author{
Alane N. Bezerra, ${ }^{1, \star}$ Natália S. Carvalho, ${ }^{2}$. Ana Carolina C. Viana, ${ }^{3}$ Suelyne R. Morais ${ }^{3}$
}

\begin{abstract}
Resumo
Devido à importância de se tratar o diabetes mellitus e prevenir suas complicações, o objetivo dessa revisão sistemática foi verificar os efeitos da suplementação de probióticos no tratamento do paciente diabético e sua contribuição na prevenção de suas complicações. Foram utilizadas as plataformas de pesquisa PubMed, Science Direct, Lilacs e Medline. Foram encontrados 11 ensaios clínicos. As principais cepas utilizadas foram dos gêneros Lactobacillus e Bifidobacterium. Os principais efeitos da suplementação de probióticos foram redução estatisticamente significativa da glicemia de jejum, da hemoglobina glicada, da frutosamina e da resistência à insulina, dos níveis séricos de colesterol total e de LDL-C, enquanto o de HDL-C aumentou. A suplementação de probióticos parece melhorar o controle glicêmico e metabólico de pacientes diabéticos.
\end{abstract}

Descritores: Probióticos; Diabetes Mellitus; Lactobacillus; Suplementação Alimentar; Complicações do Diabetes.

\section{Abstract \\ Effect of probiotics supplementation in diabetes mellitus: A systematic review}

Due to the importance of treating diabetes mellitus and prevent its complications, the aim of this systematic review was to evaluate the effects of probiotic supplementation in treatment of diabetic patient and their contribution to the prevention of its complications. Research platforms were used PubMed, Science Direct, Lilacs and Medline. 11 clinical trials were found. The main strains used were of Lactobacillus and Bifidobacterium. The main effects of supplementation of probiotics were reduction statistically significant of fasting blood glucose, glycated hemoglobin, fructosamine and insulin resistance, serum total cholesterol and LDL-C, while the HDL- C increased. Supplementation of probiotics appears to improve glycemic and metabolic control in diabetic patients.

Keywords: Probiotics; Diabetes Mellitus; Lactobacillus; Supplementary Feeding; Diabetes Complications.
1. Curso de Nutrição. Faculdade Metropolitana da Grande Fortaleza. Fortaleza, CE, Brasil.

2. Curso de Nutrição. Universidade de Fortaleza. Fortaleza, CE, Brasil.

3. Programa de Pós-Gradução. Mestrado Acadêmico de Nutrição e Saúde. Universidade Estadual do Ceará. Fortaleza, CE, Brasil.

*Endereço para correspondência:

Avenida General Osório de Paiva, 857, ap. 1003, bl. B. Fortaleza, Ceará, Brasil. CEP: 60.720-000.

E-mail: alane.nogueira@hotmail.com

Revista HUPE, Rio de Janeiro, 2016;15(2):129-139

doi: $10.12957 /$ rhupe.2016.28238

Recebido em 23/05/2016. Aprovado em 08/11/2016.

\section{Resumen}

Efecto de la suplementación con probióticos en la diabetes mellitus: una revisión sistemática

Debido a la importancia de tratarse la diabetes mellitus y prevenir sus complicaciones, el objetivo de esta revisión sistemática fue el de verificar los efectos de la suplementación con probióticos en el tratamiento de pacientes diabéticos y su contribución en la prevención de sus complicaciones. Se utilizaron las plataformas de investigación PubMed, Science Direct, Lilacs y Medline. Se encontraron 11 ensayos clínicos. Las principales cepas utilizadas fueron Lactobacillus y Bifidobacterium. Los principales efectos de la suplementación con probióticos fueron la reducción estadísticamente significativa de la glucemia en ayunas, de la hemoglobina glicosilada y fructosamina, de la resistencia a la insulina, de los niveles séricos de colesterol total y de LDL-C mientras que el HDL-C aumentó. La suplementación de probióticos parece mejorar el control glucémico y metabólico en pacientes diabéticos.

Palabras clave: Probióticos; Diabetes mellitus; Lactobacillus; Alimentación suplementaria; Complicaciones de la diabetes. 


\section{Artigo de revisão}

\section{Introdução}

O diabetes mellitus (DM) é considerado um grupo heterogêneo de distúrbios metabólicos que apresentam a hiperglicemia como característica comum e que resulta de defeitos na ação da insulina, na sua secreção ou em ambas. ${ }^{1}$ Sua prevalência vem aumentando mundialmente, observando-se uma incidência maior em mulheres do que em homens, apesar de todas as faixas etárias serem afetadas pela doença. ${ }^{2}$ No Brasil, dados de 2011 mostram que a mortalidade por DM foi de 57.853 óbitos e apresenta acentuado aumento com a progressão da idade, sendo a faixa etária de 60 anos ou mais a de maior mortalidade (80\%). Em âmbito nacional, há uma maior mortalidade no sexo feminino. ${ }^{3}$

Quando o controle da glicemia é inadequado, pode ocasionar complicações indesejáveis, como nefropatia, neuropatia e retinopatia diabética, além de outras condições patogênicas, como dislipidemia, disfunção vascular, estresse oxidativo, inflamação subclínica e alterações em vias de sinalização.,4 Paralelamente ao tratamento da hiperglicemia, é importante pensar em estratégias para a prevenção dessas complicações, devido ao risco potencial de desenvolvê-las, ${ }^{1}$ porém os tratamentos médicos atuais são ineficazes no controle dessas complicações em longo prazo. ${ }^{4}$

Com a necessidade do desenvolvimento de estratégias complementares, junto das abordagens já estabelecidas, há algumas estratégias nutricionais que podem atenuar a hiperglicemia, melhorar a função das células $\beta$ pancreáticas, a secreção de insulina, a resistência à insulina, regular o metabolismo do lipídio e das lipoproteínas, modular o estresse oxidativo e os processos inflamatórios, além de melhorar o manejo do peso corporal e prevenir complicações micro e macrovasculares. ${ }^{4}$

Uma revisão mostrou que a microbiota presente em indivíduos com DM tipo 2 parece ter relação com o desenvolvimento de complicações, como a retinopatia diabética, a toxicidade renal, a aterosclerose, a hipertensão arterial, as úlceras do pé diabético, a fibrose cística e até a doença de Alzheimer. Como a função da microbiota em manter a integridade da barreira intestinal leva à normalidade da homeostase metabólica, protegendo contra infecções e melhorando o sistema de defesa do hospedeiro, ${ }^{5}$ a modificação dessa microbiota pode ser uma nova estratégia de tratamento nas doenças metabólicas, como o DM. Essa modificação pode ocorrer por meio do uso de probióticos, por exemplo, que são preparações de células microbianas ou de seus componentes que exerçam um efeito benéfico sobre a saúde e o bem-estar do hospedeiro, ${ }^{6,7}$

Estudos experimentais mostram um possível efeito benéfico do uso de probióticos na prevenção e no tratamento do DM, possivelmente por meio da modulação da microbiota intestinal, da resposta imune e de outros mecanismos. Contudo, verificar a eficácia do uso de probióticos no controle glicêmico de indivíduos diabéticos é complexo, devido às variáveis de confusão como alimentação, uso de fármacos, composição corporal, dentre outros fatores que afetam a microbiota intestinal, o metabolismo dos carboidratos, a secreção de insulina, o balanço energético e outros hormônios

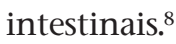

Assim, diante da importância de se prevenirem complicações crônicas associadas ao DM e da necessidade de consolidar novas estratégias terapêuticas que beneficiem o paciente diabético pela melhoria do controle glicêmico, do perfil lipídico e do estresse oxidativo, o objetivo deste artigo foi verificar os efeitos da suplementação de probióticos no tratamento do paciente diabético e sua possível contribuição na prevenção de suas complicações por meio de uma revisão sistemática de ensaios clínicos. A hipótese foi que o uso de probióticos reduziria os níveis séricos de glicemia, de triglicerídeos (TG), de colesterol total (CT) e da lipoproteína de baixa densidade (LDL) de indivíduos diabéticos.

\section{Métodos}

Foram utilizadas as plataformas de pesquisa PubMed, Science Direct, Lilacs e Medline. A pesquisa utilizou a combinação dos descritores "probióticos e diabetes" e "probiotics and diabetes" e, após exclusão dos artigos em duplicata, foram encontrados 164 artigos publicados nas línguas inglesa e portuguesa, entre os anos 2006 e 2016. Foram ainda excluídos 63 estudos com animais e 52 artigos de revisão. Após análise do título e do resumo/abstract, 38 artigos foram excluídos por não se adequarem ao tema proposto. Foram incluídos apenas ensaios clínicos que avaliaram o efeito da suplementação de probióticos em pacientes diabéticos, permanecendo 11 estudos a serem analisados nesta revisão sistemática.

Para elaboração do artigo, utilizou-se o fluxograma do método PRISMA, conforme figura 1. Para avaliar a qualidade metodológica dos 11 ensaios clínicos, utilizou-se o CONSORT 2010 checklist e cada um dos 25 itens recebeu a pontuação "zero", para o item não descrito, ou "um", para o item descrito. A pontuação total foi transformada em percentual. Foi considerado 
um ensaio clínico randomizado aquele que obteve uma pontuação maior ou igual a 70\%, moderada qualidade com pontuação entre $40 \%$ e $70 \%$ e baixa qualidade, menor ou igual a $40 \%$.

\section{Resultados e discussão}

Foram analisados 11 artigos de ensaios clínicos que verificaram os efeitos da suplementação de probióticos em indivíduos diabéticos, descritos na tabela 1. Dentre os 11 estudos, apenas 1 não era ensaio clínico duplo-cego randomizado. ${ }^{9}$ A amostra variou de 34 a 115 participantes e era composta de indivíduos com DM tipo 2 ou gestacional. As cepas utilizadas foram dos gêneros Lactobacillus, Bifidobacterium e Streptococcus, predominando os dois primeiros. Os Lactobacillus foram utilizados em todos os estudos. O tempo de intervenção variou de 4 a 12 semanas.

Razmpoosh e colaboradores ${ }^{10}$ observaram que o gênero bacteriano mais utilizado pelos estudos foi o Lactobacillus. Essas cepas apresentaram papel positivo na melhoria da hiperglicemia tanto a curto como a longo prazo, evidenciando reduções na glicemia de jejum e na pós-prandial, na hemoglobina glicada (HbA1c), nas concentrações séricas de insulina e na resistência à insulina.

Os principais efeitos benéficos da suplementação de probióticos em indivíduos diabéticos tipo 2 foram redução estatisticamente significativa da glicemia de jejum, ${ }^{11,12,13}$ da HbA1c11,13,14,15 e da frutosamina. ${ }^{15}$ Outros achados, embora não significativos, podem reforçar o

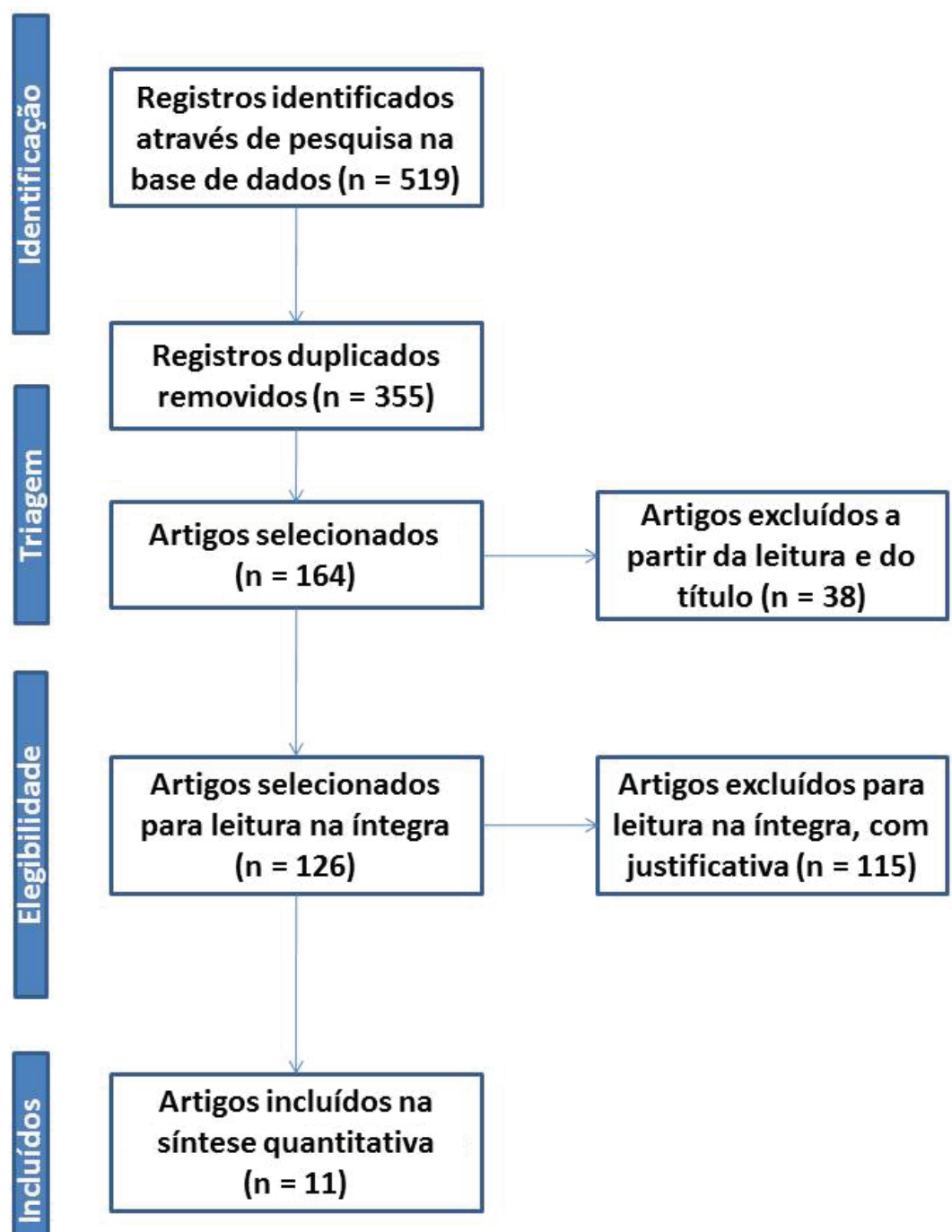

Figura 1. Fluxograma da seleção de artigos para a revisão. Método PRISMA. 


\section{Artigo de revisão}

\begin{tabular}{|c|c|c|c|}
\hline 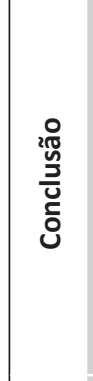 & 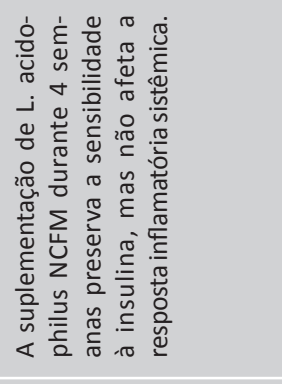 & 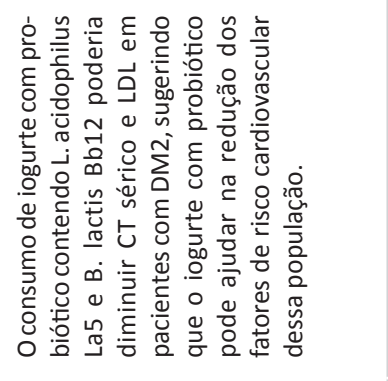 & 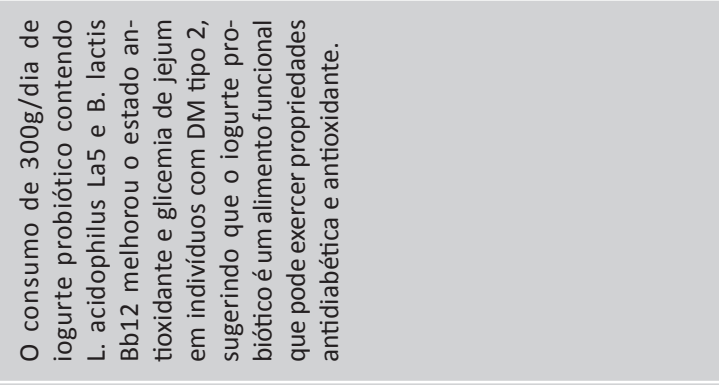 \\
\hline 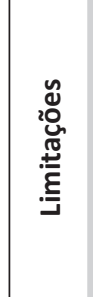 & 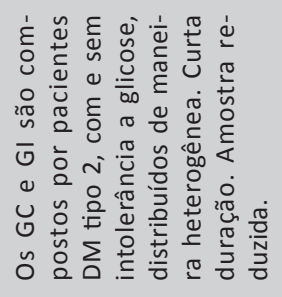 & 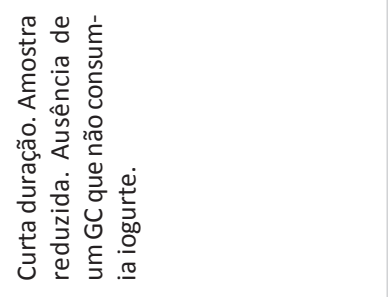 & 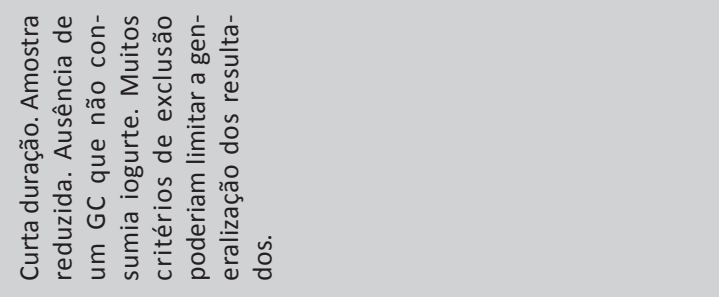 \\
\hline 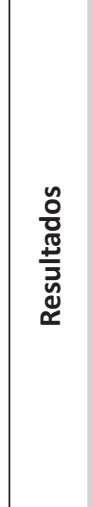 & 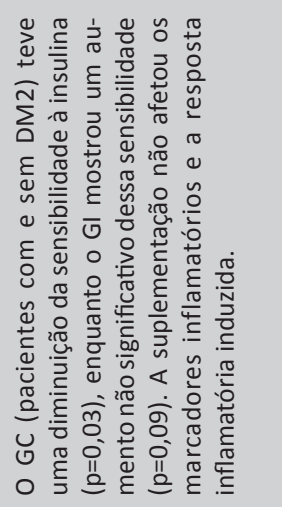 & 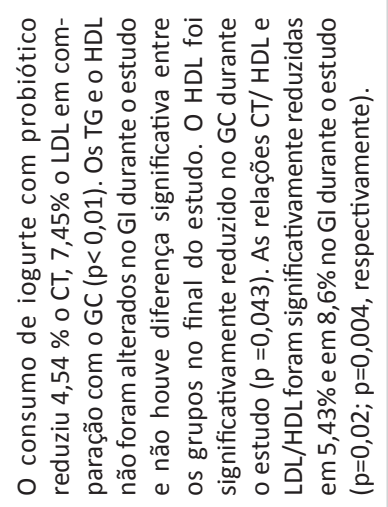 & 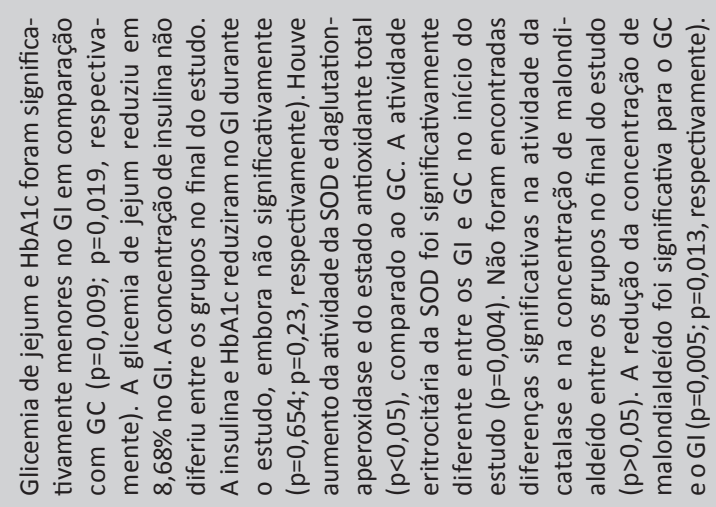 \\
\hline $\begin{array}{l}\stackrel{0}{0} \\
\stackrel{0}{0} \\
\frac{0}{\partial} \\
\end{array}$ & 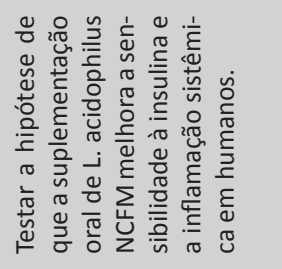 & 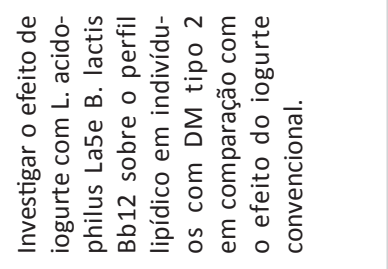 & 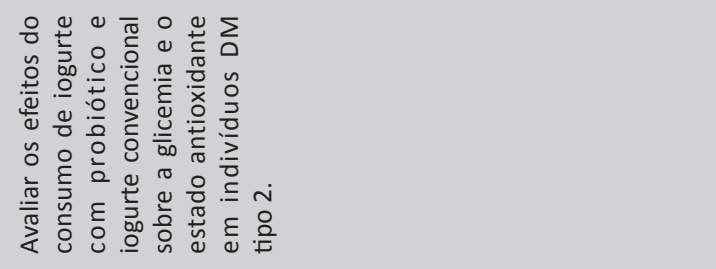 \\
\hline 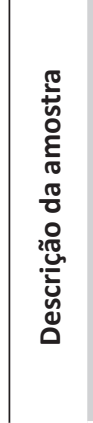 & 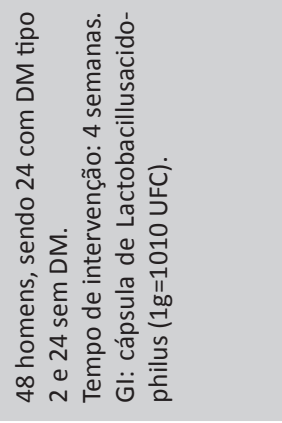 & 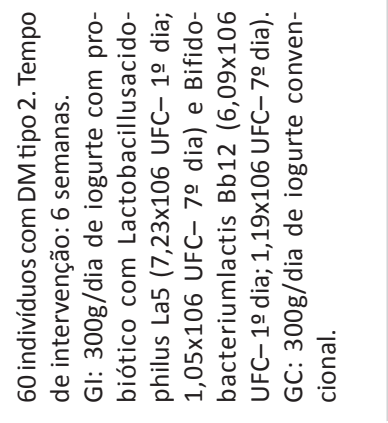 & 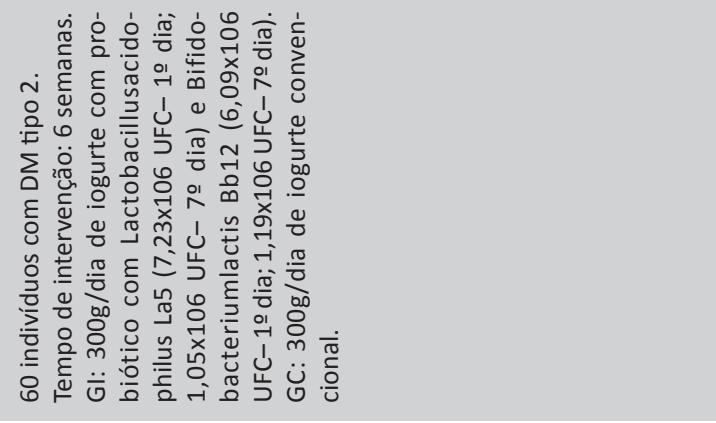 \\
\hline 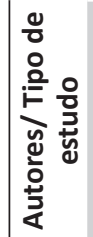 & 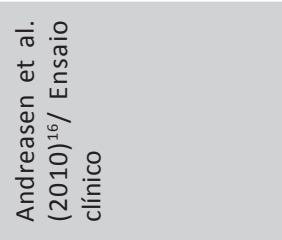 & 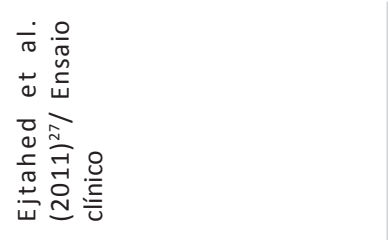 & 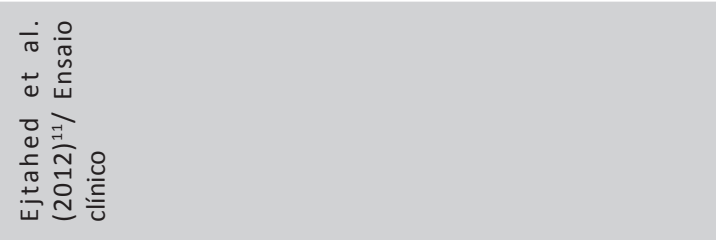 \\
\hline
\end{tabular}



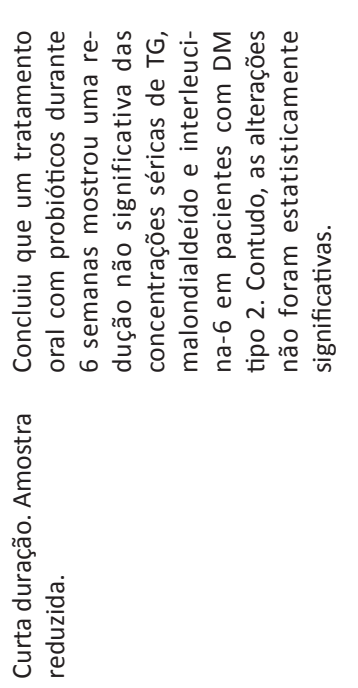

نิ่

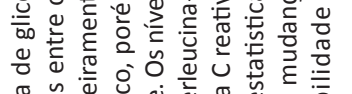

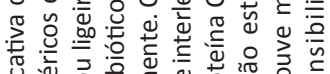

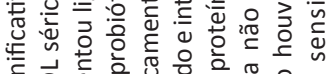

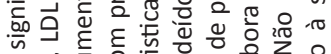

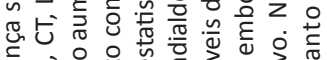

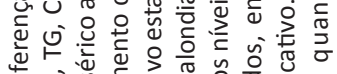

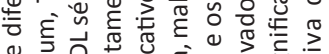

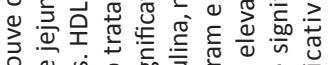

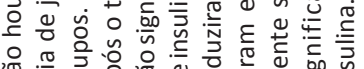

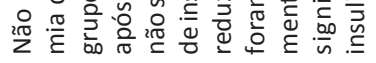

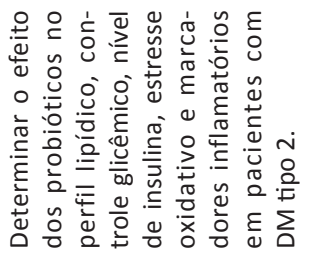
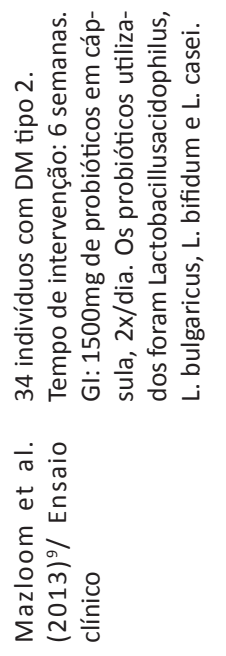
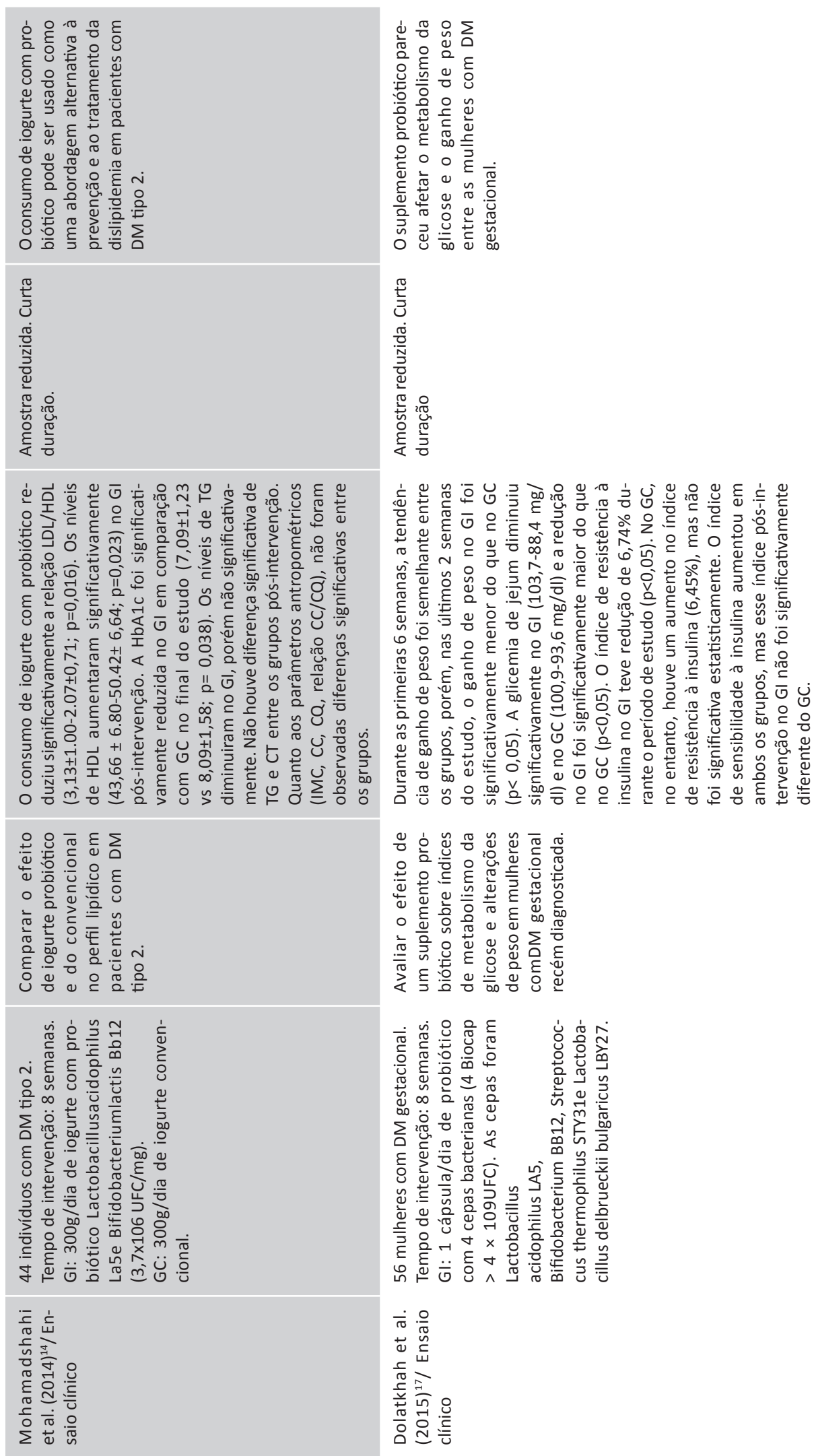


\section{Artigo de revisão}

\begin{tabular}{|c|c|c|}
\hline 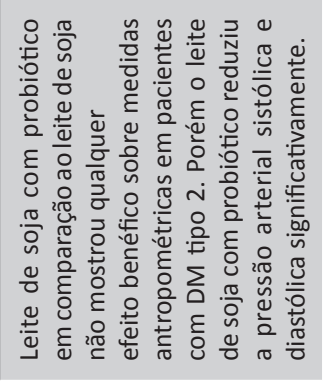 & 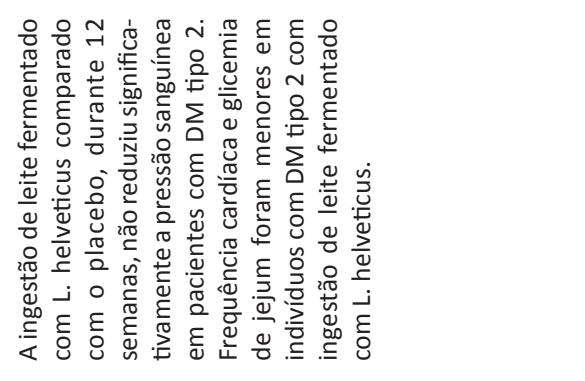 & 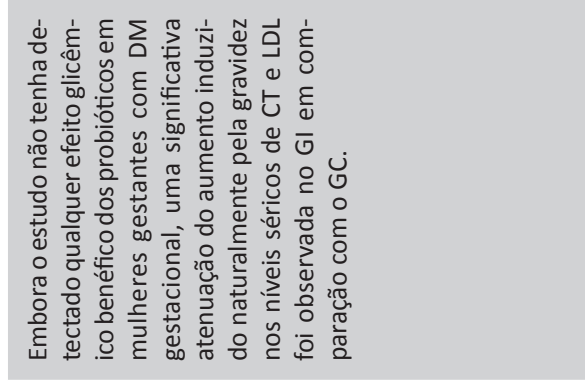 \\
\hline 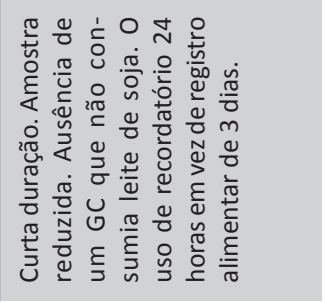 & 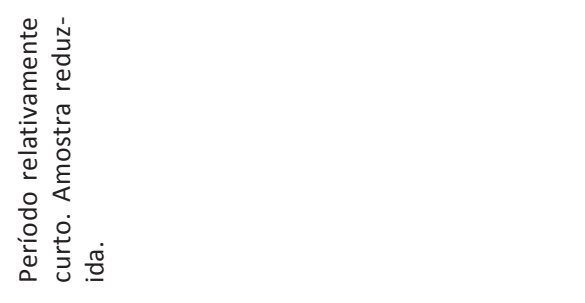 & 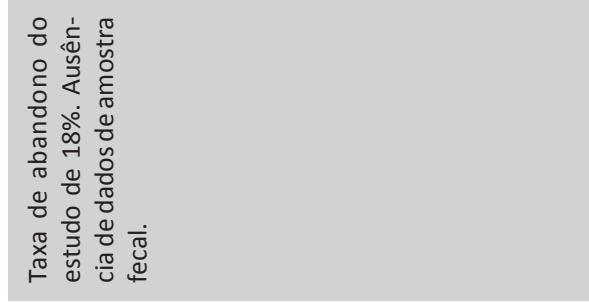 \\
\hline 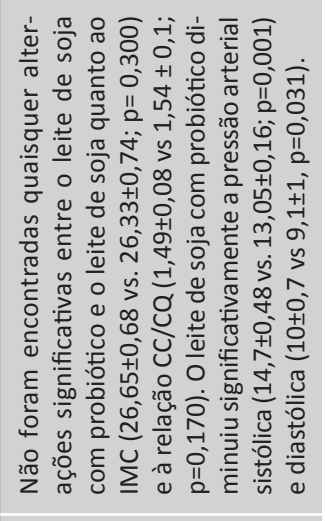 & 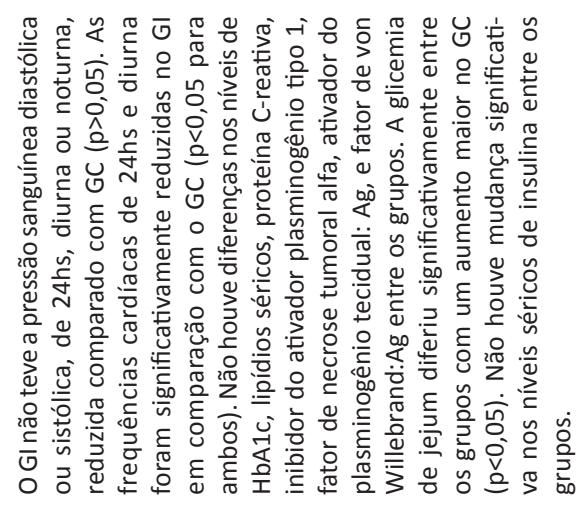 & 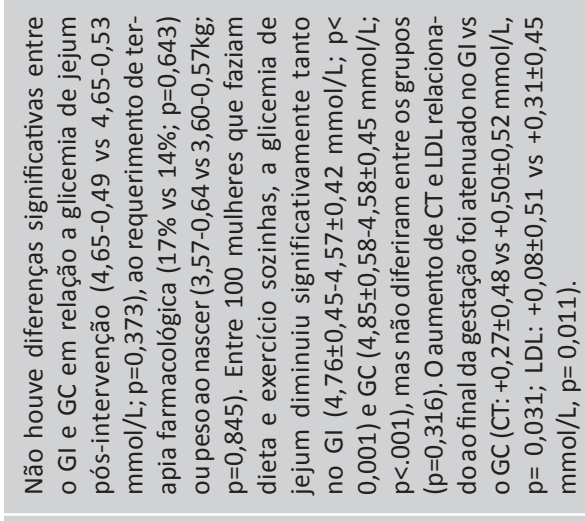 \\
\hline 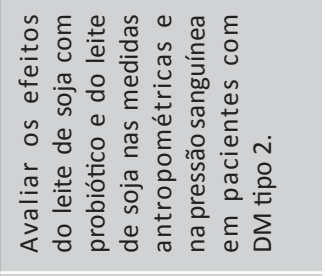 & 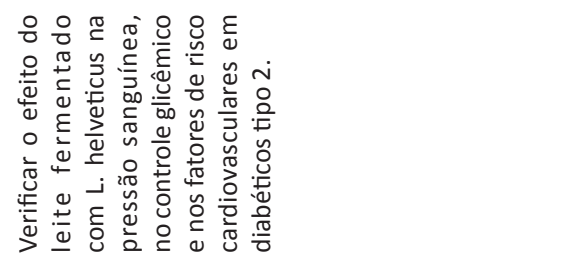 & 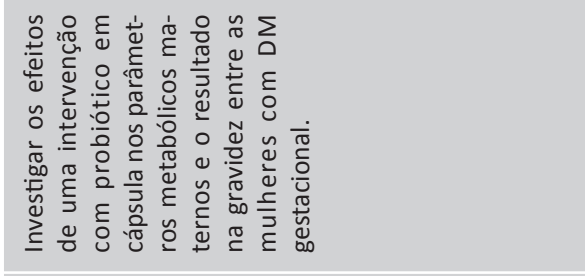 \\
\hline 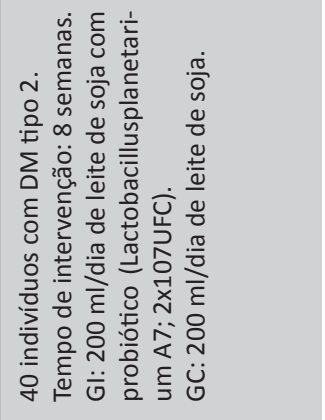 & 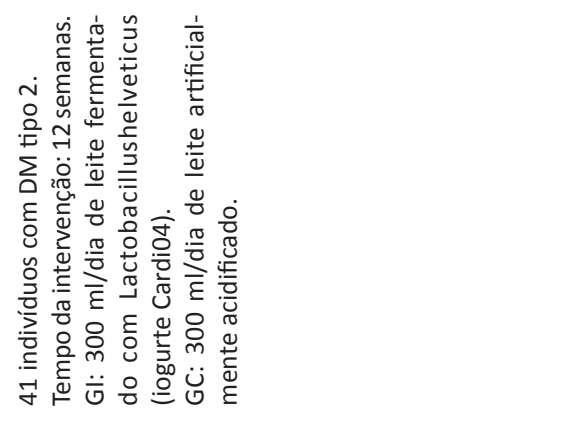 & 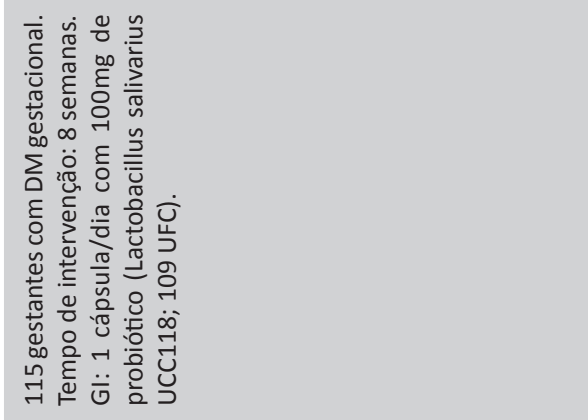 \\
\hline 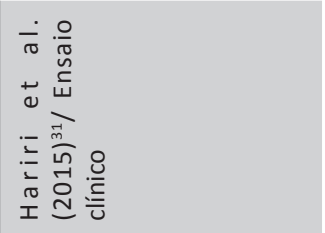 & 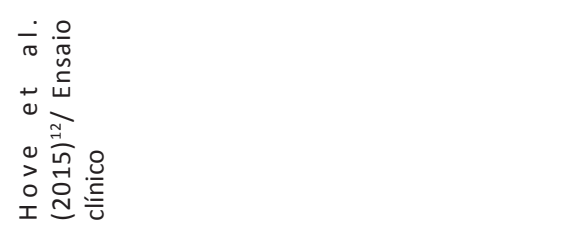 & 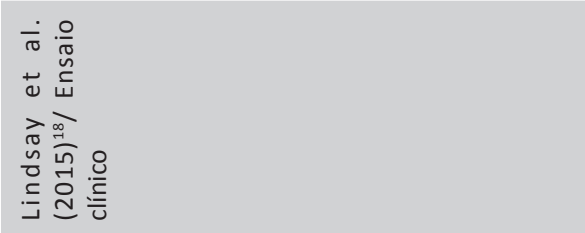 \\
\hline
\end{tabular}



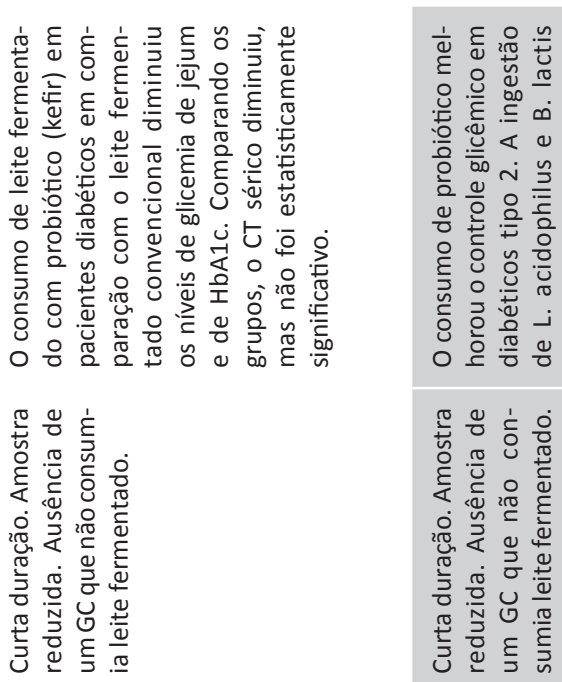

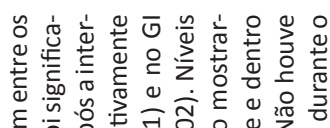

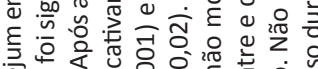

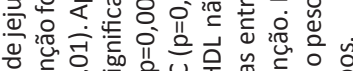

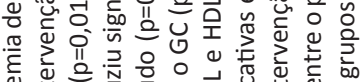

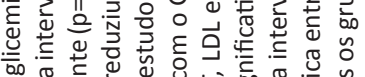

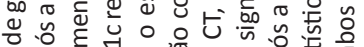

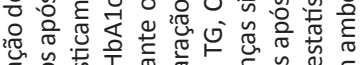
嵌

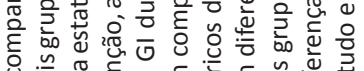

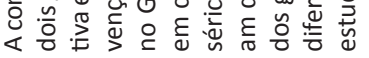

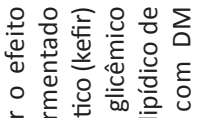

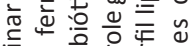

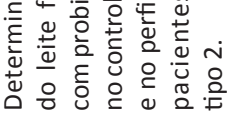

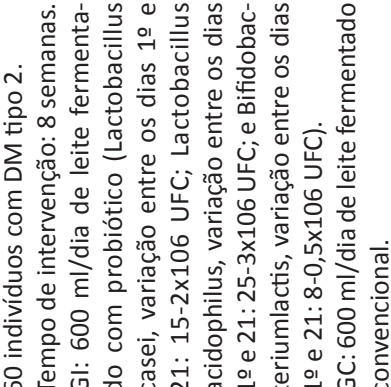

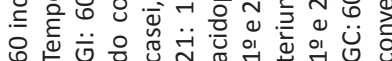

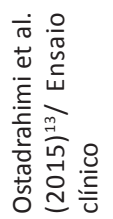

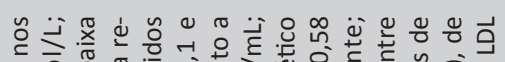

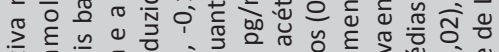

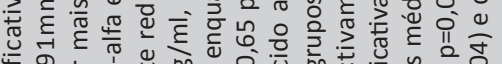
ò

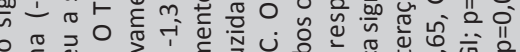
20.

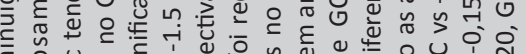

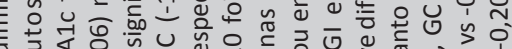

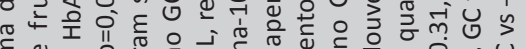

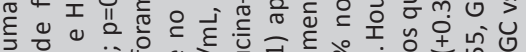

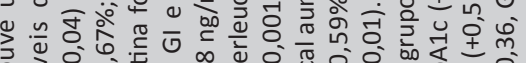

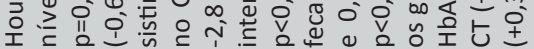
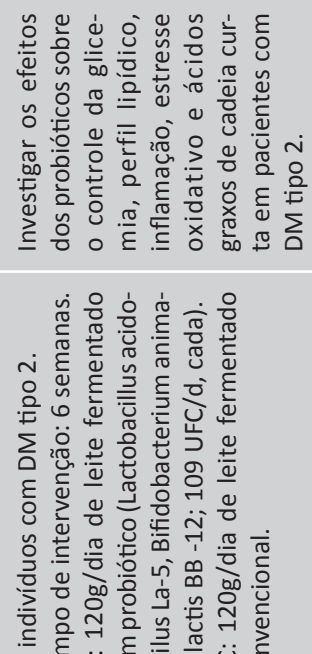

守灾记

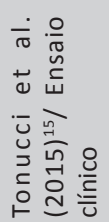

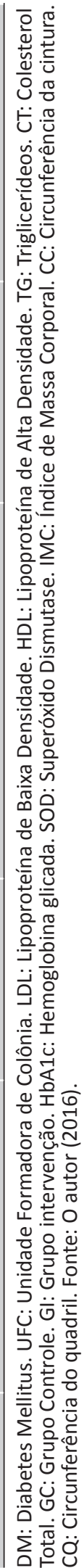




\section{Artigo de revisão}

possível efeito benéfico da suplementação dos probióticos sobre o controle glicêmico, como a redução da $\mathrm{HbA}_{1} \mathrm{c}^{11}$ e dos níveis séricos de insulina, ${ }^{9,11}$ enquanto a sensibilidade à insulina aumentou. ${ }^{16}$

Dentre os estudos com DM gestacional, ${ }^{17,18}$ foram verificadas reduções significativas da glicemia de jejum e da resistência à insulina e uma tendência, embora não significativa, de aumento da sensibilidade à insulina nessas gestantes. ${ }^{17}$

Uma meta-análise de ensaios clínicos com indivíduos com DM tipo 2 verificou que o consumo de probióticos leva a uma redução na HbA1c e nos níveis de glicemia de jejum. Os melhores resultados foram observados em intervenções com duração maior que 8 semanas e com a utilização de várias cepas de probiótico. Além disso, essa terapia nutricional auxiliou na redução da resistência à insulina e de seus níveis séricos. Isso revela a importância dos probióticos na otimização do metabolismo da glicose. ${ }^{19}$

A utilização de Lactobacillus gasseri BNR17, proveniente do leite materno humano, reduziu significativamente a glicemia de jejum e a pós-prandial e, embora não significativo, a HbA1c. ${ }^{20} \mathrm{O}$ uso de Lactobacillus rhamnosus CCFM0528 e o de Lactobacillus casei CCFM 0412 também reduziram a glicemia de jejum, a pós-prandial e a HbA1c, sendo os níveis séricos de insulina e o glicogênio hepático aumentados após a intervenção por 13 semanas em ratos diabéticos, mostrando uma melhoria na tolerância à glicose. .11,22 Outro estudo experimental com ratos diabéticos usando leite de soja fermentado com Lactobacillus rhamnosus CRL 981 demonstrou redução significativa na glicemia de jejum. ${ }^{23} \mathrm{O}$ uso das cepas bacterianas Lactobacillus acidophilus e Lactobacillus casei também promoveram redução da glicosilação da hemoglobina. ${ }^{24}$

O uso de shubat, uma mistura com cepas bacterianas produtoras de ácido lático (Lactobacillus plantarum, L. helveticus, L. harbinensis, L. hilgardii, L. rhamnosus, L. mucosae, L. par, L. paracasei subsp. tolerans, L. pentosus e Lactococcus lactis) e leveduras (Kluyveromyce smarxianus, Pichia membranifaciens, Candida ethanolica e Issatchenkia orientalis), promoveu a redução da glicemia de jejum e da HbA1c e o aumento dos níveis séricos de peptídeo-Ce do peptídeo semelhante ao glucagon (GLP-1). ${ }^{25}$ Ainda, uma meta-análise verificou que o uso de probióticos em diabéticos tipo 2 reduziu significativamente os níveis de $\mathrm{HbA1c}$ e a resistência à insulina, embora essa redução não tenha se mostrado significativa sobre a glicemia de jejum e os níveis séricos de insulina. ${ }^{26}$
Quanto ao perfil lipídico, os níveis séricos de CT e de LDL reduziram, ${ }^{15,27}$ enquanto o de HDL aumentou em indivíduos diabéticos tipo $2 .{ }^{14}$ Ainda que não significativo, os estudos mostraram redução dos níveis séricos de $\mathrm{TG}^{9,14}$ e aumento dos níveis séricos de HDL nos grupos de intervenção. ${ }^{9}$

O uso de leite fermentado com Bifidobacterium lactis HN019, durante 45 dias, reduziu os níveis séricos de CT e de LDL, sugerindo uma melhora do perfil lipídico. ${ }^{28}$ Um estudo experimental com ratos diabéticos usando leite de soja fermentado com Lactobacillus rhamnosus CRL 981 demonstrou uma redução significativa nos níveis séricos de $\mathrm{CT} .{ }^{23} \mathrm{O}$ uso de Lactobacillus casei CCFM 0412 verificou redução significativa dos níveis sanguíneos dos TG, CT e LDL e aumentou o HDL em 13 semanas em ratos com DM tipo 2.22 Já a suplementação da cepa bacteriana Lactobacillus plantarium TN627 reverteu a atividade das lípases pancreática e sérica em ratos diabéticos, levando à redução dos TG, LDL e ao aumento do HDL. ${ }^{29}$

O leite de soja com Lactobacillus plantarum A7 KC 355240 proporcionou um aumento significativo de HDL em ratos diabéticos, o que sugere um efeito modulador dessa cepa, auxiliando na redução dos riscos de aterosclerose e de complicações associadas ao DM. ${ }^{30}$ Entretanto, uma meta-análise não observou aumento significativo de HDL em pacientes diabéticos tipo 2, possivelmente devido a heterogeneidade dos estudos analisados. ${ }^{26}$

A dislipidemia é um fator de risco da progressão do DM tipo 2 e o uso de probiótico auxilia na modulação do perfil lipídico. ${ }^{10}$ Uma meta-análise sugere uma ação benéfica dos probióticos sobre alguns fatores de risco cardiometabólicos em diabéticos tipo 2. Observou-se uma redução não significativa dos níveis séricos de proteína-C reativa, de CT, de TG e de LDL. ${ }^{26}$

Além disso, a pressão arterial sistêmica ${ }^{31}$ e a frequência cardíaca ${ }^{12}$ diminuíram significativamente. Os probióticos exibem um potencial efeito anti-hipertensivo por meio da melhoria do perfil lipídico, da resistência à insulina e de outras vias metabólicas, o que reporta seu uso como estratégia nutricional na prevenção e no tratamento de diabéticos, reduzindo os riscos de desenvolvimento de hipertensão arterial associada ao DM. ${ }^{32}$ Além de atuar nesta comorbidade, tem um potencial preventivo e de redução da gravidade do DM tipo 2 e de outras síndromes metabólicas, possivelmente, pela modulação da microbiota intestinal e da resposta imune, dentre outros mecanismos. ${ }^{8}$

Embora não significativo, foi verificada uma 
redução dos marcadores inflamatórios. ${ }^{9} \mathrm{O}$ uso do probiótico Lactobacillus casei Shirota proporcionou uma redução significativa dos níveis séricos de citocinas pró-inflamatória (Proteína-C reativa, interleucina-6) e de neutrófilos, mostrando uma ação moduladora dos processos inflamatórios e, consequentemente, uma redução sugestiva do risco de desenvolver complicações diabéticas..$^{33}$ O Lactobacillus rhamnosus CCFM0528 reduziu o TNF-alfa, a interleucina-6 e a interleucina-8, sendo os níveis de interleucina-4 e de interleucina-10 aumentados após a intervenção por 13 semanas em ratos diabéticos. ${ }^{21}$

O TNF-alfa e a resistina reduziram durante o período de intervenção de apenas um estudo analisado na tabela $1 .{ }^{15} \mathrm{O}$ uso de Bifidobacterium lactis HNO19 por 45 dias reduziu os níveis séricos de citocinas pró-inflamatórias, como o TNF-alfa e a interleucina-6, sugerindo uma redução da obesidade e de alguns marcadores inflamatórios. Por redução de risco cardiovascular, esse probiótico pode beneficiar os indivíduos com síndrome metabólica, como o diabético com complicações associadas. ${ }^{28}$

A alimentação pode influenciar na composição da microbiota do intestino e aumentar a quantidade de bactérias gram negativas, podendo levar a uma maior permeabilidade intestinal. Isso pode levar a translocação de lipopolissacarídeos do lúmen intestinal para a circulação sanguínea, provocando respostas inflamatórias, como a liberação de citocinas (TNF-alfa e interleucina-6) e o aumento do estresse oxidativo. Como consequência, pode-se desenvolver uma resistência à insulina, por sua sinalização diminuída. Além disso, a microbiota pode reduzir a secreção intestinal do GLP-1, levando a uma menor sensibilidade à insulina. Já que a composição da microbiota intestinal pode influenciar diversas vias metabólicas e é passível de ser modificada pela alimentação, estratégias nutricionais como o uso de probióticos podem beneficiar o hospedeiro. ${ }^{8}$

Chen e colaboradore ${ }^{22}$ verificaram redução significativa dos níveis séricos do TNF-alfa, de malondialdeído e de espécies reativas de oxigênio em ratos com DM tipo 2 em uso de Lactobacillus casei CCFM 0412. Quanto às concentrações séricas de interleucina-10, peróxidos de glutationa e superóxido dismutase, aumentaram de forma significativa em 13 semanas, mostrando uma melhoria nas propriedades imunorregulatórias e no estresse oxidativo. ${ }^{21}$

Oleite de soja fermentado com Lactobacillus rhamnosus CRL 981tambémevidenciou um aumento na atividade da catalase hepática e da superóxido dismutase em ratos diabéticos, mostrando uma possível melhoria em relação ao estresse oxidativo. ${ }^{23} \mathrm{~A}$ enzima superóxido dismutase também teve sua atividade aumentada em ratos diabéticos sob o uso de probióticos com Lactobacillus acidophilus, Bifidobacterium lactis e Lactobacillus fermentum..$^{34} \mathrm{O}$ uso de probiótico contendo Lactobacillus acidophilus e Lactobacillus casei reduziu as espécies reativas do ácido tiobarbitúrico, marcador de estresse oxidativo no intestino, além de promover um equilíbrio microbiano intestinal e uma redução do tempo de trânsito intestinal em ratos diabéticos. ${ }^{24}$

O ganho de peso gestacional foi menor estatisticamente em gestantes com DM gestacional usando probióticos. ${ }^{17} \mathrm{O}$ uso do Saccharomyces boulardii em camudongos diabéticos mostrou uma redução do peso corporal, da massa gorda, da esteatose hepática e da inflamação, o que parece estar associado aos efeitos locais no intestino do hospedeiro, podendo ter papel importante no tratamento da obesidade e do DM. ${ }^{35}$

O uso de shubat levou a uma redução do peso corpóreo, diminuição da creatinina e ureia séricas em ratos diabéticos. Isso promove uma proteção da função renal e das ilhotas pancreáticas no DM tipo 2 e modulação do metabolismo lipídico, ${ }^{25}$, importantes na prevenção de complicações crônicas associadas ao DM.

Um estudo com japoneses diabéticos tipo 2 detectou bactérias intestinais na corrente sanguínea, quantidade significativamente maior do que em indivíduos não diabéticos, sugerindo a translocação bacteriana do intestino para o sangue. Além disso, observou-se que o conteúdo fecal de ácido acético e de ácido propiônico foram significativamente menores nos indivíduos com diabetes. Uma ingestão de ácidos graxos saturados e de gorduras totais, bem como a duração do diabetes, apresentaram correlação negativa com o conteúdo fecal de ácido acético, enquanto que o consumo de carboidratos se correlacionou positivamente, ${ }^{36}$ mostrando que a microbiota intestinal pode ser modificada com a alimentação.

O conteúdo fecal de ácido acético aumentou significativamente no único estudo que foi verificado. ${ }^{15}$ A suplementação do probiótico Lactobacillus plantarum NCU116 em ratos diabéticos durante 5 semanas aumentou a quantidade ácidos graxos de cadeia curta, como o ácido acético, propiônico e butírico, produtos finais da fermentação bacteriana do lúmen intestinal, no colón. 15,37

Quanto à qualidade metodológica dos ensaios clínicos randomizados, $6^{11,12,15,17,18,31}$ dos 11 estudos apresentaram alta qualidade metodológica, enquanto os 


\section{Artigo de revisão}

outros, $, 13,14,16,27$ moderada qualidade.

Embora os resultados não apresentem uniformidade entre os artigos discutidos, devido ao tamanho da amostra, ao tempo de duração e à variedade das cepas e da quantidade utilizada, a suplementação de probióticos parece melhorar o controle metabólico de pacientes diabéticos e prevenir e/ou auxiliar no tratamento de complicações associadas

\section{Conclusão}

A presente revisão sistemática de ensaios clínicos oferece um otimismo cauteloso quanto à suplementação de probióticos no DM. As cepas de Lactobacillus e Bifidobacterium são as mais utilizadas e a elas podem ser atribuídos os efeitos benéficos no tratamento do DM, bem como o potencial na prevenção e/ou no tratamento de complicações associadas. A suplementação de probióticos em diabéticos auxilia na redução estatisticamente significativa da glicemia de jejum, da hemoglobina glicada, da frutosamina e da resistência à insulina, e na melhora do perfil lipídico, diminuindo os níveis séricos de colesterol total e de LDL-C e aumentando o de HDL-C. No entanto, existe a necessidade de mais ensaios clínicos randomizados com padronizações de medidas, como amostra e período de duração maiores, a fim de determinar as cepas dos probióticos, a duração e a dose da suplementação, para verificar e/ou confirmar com maior evidência os resultados descritos pelos estudos.

\section{Referências}

1. Sociedade Brasileira de Diabetes (SBD). Diretrizes da Sociedade Brasileira de Diabetes: 2014/2015. São Paulo: AC Farmacêutica; 2015.

2. Tao Z, Shi A, Zhao J. Epidemiological perspectives of diabetes. Cell BiochemBiophys 2015; 73(1):1-5

3. Brasil. Ministério da Saúde. Secretaria de Vigilância em Saúde. Sistema de Informações sobre Mortalidade. Disponível em: http://tabnet.datasus.gov.br/cgi/ deftohtm.exe?idb2012/c12. def. Acessado em 20/02/2016.

4. Mirmiran P, Bahadoran Z, Azizi F. Functional foods-based diet as a novel dietary approach for management of type 2 diabetes and its complications: A review. World J Diabetes 2014;5(3):267-81.

5. Zhang Y, Zhang H. Microbiota associated with type 2 diabetes and its related complications. Food Sci Hum Wellness. 2013;2(3):167-72.

6. Esteve E, Ricart W, Fernandez-Real J. Gut microbiota interactions with obesity, insulin resistance and type 2 diabetes: did gut microbiote co-evolve with insulin resistance?.Curr Opin Clin Nutr Metab Care. 2011;14(5):483-90.

7. Salminen S, Ouwehand A, Benno Y, et al. Probiotics: how should they be defined?.Trends Food Sci Technol. 1999;10(3):107-10.

8. Tonucci LB, Santos KO, Ferreira CLLF, et al. Clinical Applica- tion of Probiotics in Diabetes Mellitus: Therapeutics and New Perspectives.Crit Rev Food SciNutr. 2015.

9. Mazloom Z, Yousefinejad A, Dabbaghmanesh MH. Effect of probiotics on lipid profile, glycemic control, insulin action, oxidative stress, and inflammatory markers in patients with type 2 diabetes: a clinical trial. Iran J Med Sci 2013;38(1):38-43.

10. Razmpoosh E, Javadi M, Ejtahed HS, et al. Probiotics as beneficial agents in the management of diabetes mellitus: a systematic review. Diabetes Metab Res Rev 2015;32:143-68.

11. Ejtahed HS, Mohtadi-Nia J, Homayouni-Rad A, et al. Probiotic yogurt improves antioxidant status in type 2 diabetic patients. Nutrition. 2012;28(5): 539-43.

12. Hove KD, Brøns C, Færch K, et al. Effects of 12 weeks of treatment with fermented milk on blood pressure, glucose metabolism and markers of cardiovascular risk in patients with type 2 diabetes: a randomised double-blind placebo-controlled study. Eur J Endocrinol. 2015;172(1):11-20.

13. Ostadrahimi A, Taghizadeh A, Mobasseri M, et al. Effect of probiotic fermented milk (kefir) on glycemic control and lipid profile in type 2 diabetic patients: a randomized double-blind placebo-controlled clinical trial. Iran J Public Health. 2015; 44(2): 228-37.

14. Mohamadshahi M, Veissi M, Haidari F, et al. Effects of probiotic yogurt consumption on lipid profile in type 2 diabetic patients: A randomized controlled clinical trial. J Res Med Sci. 2014;19(6):531-6.

15. Tonucci LB, Santos KMO, Oliveira LL, et al. Clinical application of probiotics in type 2 diabetes mellitus: A randomized, double-blind, placebo-controlled study. ClinNutr. 2015;1-8.

16. Andreasen AS, Larsen N, Pedersen-Skovsgaard T, et al. Effects of Lactobacillus acidophilus NCFM on insulin sensitivity and the systemic inflammatory response in human subjects. $\mathrm{Br} \mathrm{J}$ Nutr. 2010;104(12):1831-8.

17. Dolatkhah N, Hajifaraji M, Abbasalizadeh F, et al. Is there a value for probiotic supplements in gestational diabetes mellitus? A randomized clinical trial. J Health Popul Nutr. 2015;33(1):1-12.

18. Lindsay KL, Brennan L, Kennelly MA, et al. Impact of probiotics in women with gestational diabetes mellitus on metabolic health: a randomized controlled trial. Am J Obstet Gynecol. 2015;212(4):496-e1.

19. Zhang $Q$, Wu Y, Fei X. Effect of probiotics on glucose metabolism in patients with type 2 diabetes: A meta-analysis of randomized controlled trials. Medicina (Kaunas). 2015.

20. Yun SI, Park HO, Kang JH. Effect of Lactobacillus gasseri BNR17 on blood glucose levels and body weight in a mouse model of type 2 diabetes. J ApplMicrobiol. 2009;107(5):1681-6.

21. Chen $P$, Zhang Q, Dang $H$, et al. Oral administration of Lactobacillus rhamnosus CCFM0528 improves glucose tolerance and cytokine secretion in high-fat-fed, streptozotocin-induced type 2 diabetic mice. J Funct Foods. 2014;10:318-26.

22. Chen $P$, Zhang Q, Dang $\mathrm{H}$, et al. Antidiabetic effect of Lactobacillus casei CCFM0412 on mice with type 2 diabetes induced by a high-fat diet and streptozotocin. Nutrition. 2014;30(9):1061-8.

23. Marazza JA, LeBlanc JG, Giori GS, et al. Soymilk fermented with Lactobacillus rhamnosus CRL981 ameliorates hyperglycemia, lipid profiles and increases antioxidant enzyme activities in diabetic mice. J Funct Foods. 2013;5(4):1848-53.

24. Yadav $H$, Jain S, Sinha PR. The effect of probiotic dahi containing Lactobacillus acidophilus and Lactobacillus casei on gastropathic consequences in diabetic rats. J Med Food. 2008;11(1):62-68.

25. Manaer T, Yu L, Zhang Y, et al. Anti-diabetic effects of shubat in type 2 diabetic rats induced by combination of high-glucose-fat diet and low-dose streptozotocin. J Ethnopharmacol. 
Alane N. Bezerra e cols. • Efeito da suplementação de probióticos no diabetes mellitus: uma revisão sistemática

2015;169:269-74.

26. Kasinska MA, Drzewoski J. Effectiveness of probiotics in type 2 diabetes: a meta-analysis. Pol Arch Med Wewn. 2015;125:803-13.

27. Ejtahed HS, Mohtadi-Nia J, Homayouni-Rad A, et al. Effect of probiotic yogurt containing Lactobacillus acidophilus and Bifidobacterium lactis on lipid profile in individuals with type 2 diabetes mellitus. J Dairy Sci. 2011; 94(7):3288-94.

28. Bernini LJ, Simão ANC, Alfieri DF, et al. Beneficial effects of Bifidobacterium lactis on lipid profile and cytokines in patients with metabolic syndrome: a randomized trial. Nutrition. 2015.

29. Bejar W, Hamden K, Salah RB, et al. Lactobacillus plantarum TN627 significantly reduces complications of alloxan-induced diabetes in rats. Anaerobe. 2013;24:4-11.

30. Babashahi M, Mirlohi M, Ghiasvand R, et al. Comparison of soymilk and probiotic soymilk effects on serum high-density lipoprotein cholesterol and low-density lipoprotein cholesterol in diabetic Wistar rats. ARYA atheroscler. 2015;11(Suppl 1):88.

31. Hariri M, Salehi R, Feizi A, et al. The effect of probiotic soy milk and soy milk on anthropometric measures and blood pressure in patients with type II diabetes mellitus: A randomized double-blind clinical trial. ARYA Atheroscler. 2015;11(Suppl 1):74-80.
32. Lye HS, Kuan CY, Ewe JA, et al. The improvement of hypertension by probiotics: effects on cholesterol, diabetes, renin, and phytoestrogens. Int J Mol Sci. 2009;10(9):3755-75.

33. Zarfeshani A, Khaza'ai H, Ali RM, et al. Effect of Lactobacillus casei on the production of pro-inflammatory markers in streptozotocin-induced diabetic rats. Probiotics Antimicrob Proteins. 2011;3(3-4):168-74.

34. Davari S, Talaei SA, Alaei H. Probiotics treatment improves diabetes-induced impairment of synaptic activity and cognitive function: behavioral and electrophysiological proofs for microbiome-gut-brain axis. Neuroscience. 2013;240:287-96.

35. Everard A, Matamoros S, Geurts L, et al. Saccharomyces boulardii administration changes gut microbiota and reduces hepatic steatosis, low-grade inflammation, and fat mass in obese and type 2 diabetic db/db mice. M Bio. 2014;5(3): e01011-14.

36. Sato J, Kanazawa A, Ikeda F, et al. Gut dysbiosis and detection of "live gut bacteria" in blood of Japanese patients with type 2 diabetes. Diabetes Care. 2014;37(8):2343-50.

37. Li C, Ding Q, Nie SP, et al. Carrot juice fermented with Lactobacillus plantarum NCU116 ameliorates Type 2 diabetes in rats. J Agric Food Chem. 2014;62(49):11884-91. 